\title{
Supplementary Information of
}

\section{"A new systematic construction of novel 3- dimensional spin crossover coordination polymers based on $\left[\mathrm{Ag}_{2}^{\mathrm{I}}(\mathrm{CN})_{3}\right]$ building unit}

Takashi Kosone, ${ }^{* \dagger}$ Syogo Okuda $\$$, Masaya Kawata ${ }^{\dagger}$, Shunsuke Arai ${ }^{\dagger}$, Ryota Kosuge ${ }^{\dagger}$ and Takeshi Kawasaki ${ }^{\dagger \dagger}$

${ }^{\dagger}$ Department of Science and Engineering, Graduate School of Science and Engineering, Tokyo Denki University, Hatoyama, Hiki-gun, Saitama, 350-0394, Japan Tel: +81-49-296-2923; E-mail: $\underline{\text { t- }}$ kosone@mail.dendai.ac.jp

\$Department of Materials Science and Technology, Nagaoka University of Technology, 1603-1, Kamitomioka, Nagaoka, Niigata, 94-2188, Japan

†Department of Chemistry, Faculty of Science, Toho University, 2-2-1 Miyama, Funabashi, Chiba 2748510, Japan 


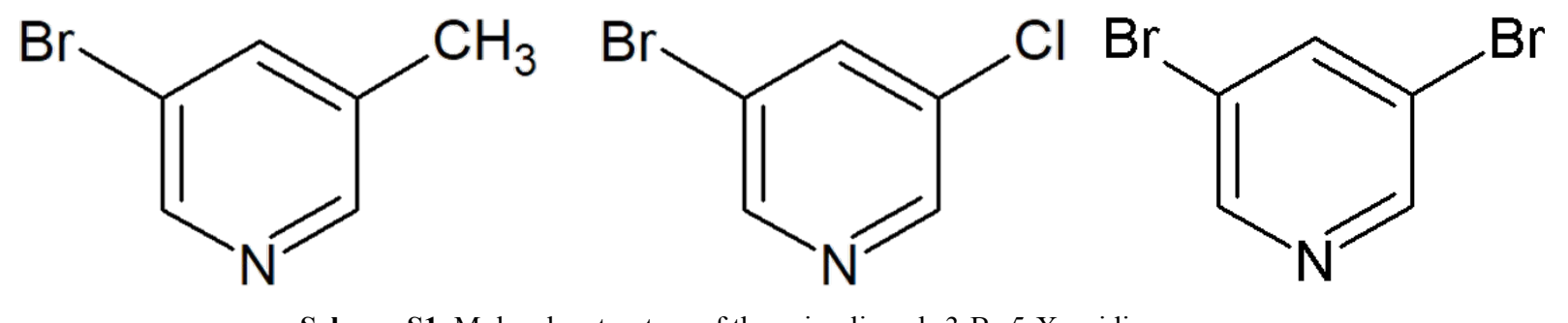

Scheme S1. Molecular structure of the using ligands 3-Br-5-Xpyridine. 


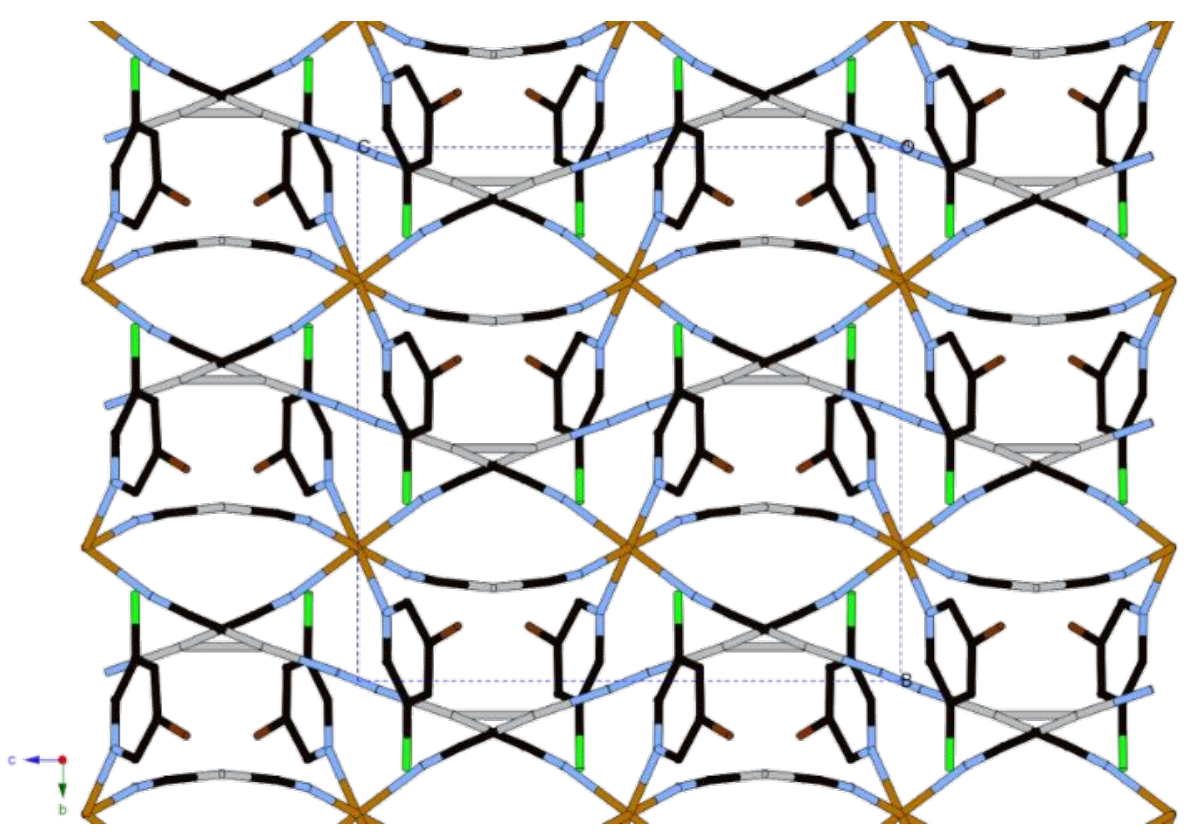

Figure S1. The crystal structure of 2 at 300K. Atom code: Fe (brown), Ag (grey), N (blue) $\mathrm{C}$ (black), $\mathrm{Br}$ (Brown) and $\mathrm{Cl}$ (yellow green). In this picture, hydrogen atoms are omitted for

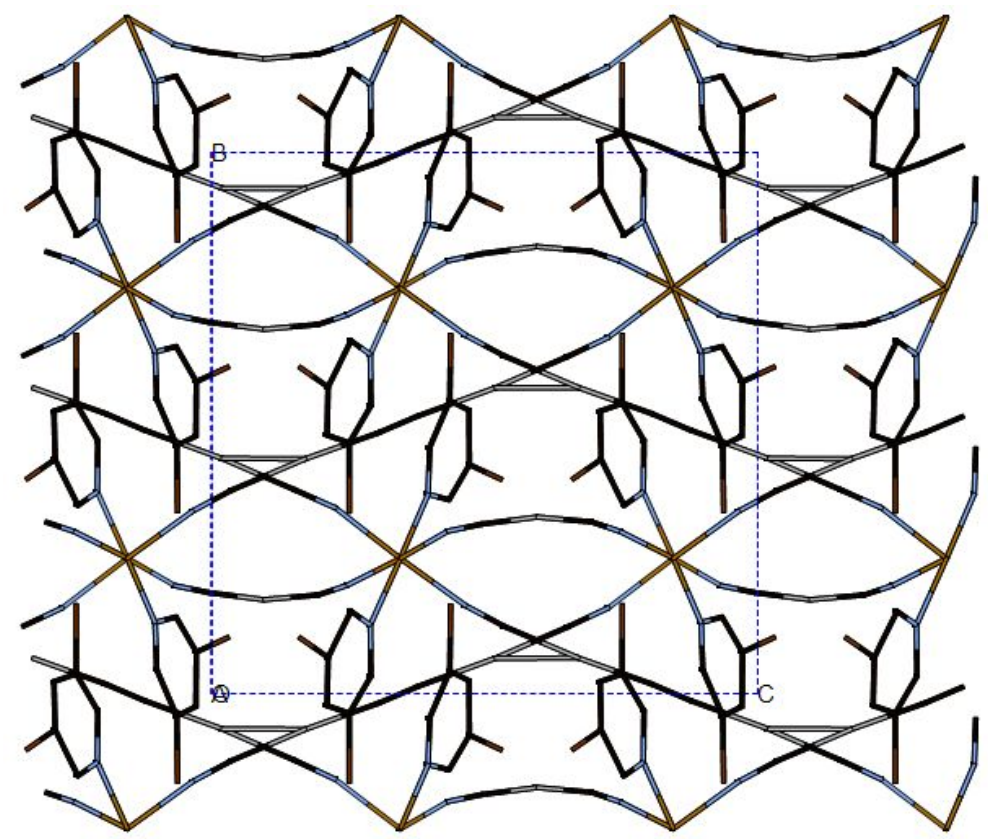

Figure S2. The crystal structure of $\mathbf{3}$ at 300K. Atom code: Fe (brown), Ag (grey), N (blue) $\mathrm{C}$ (black), $\mathrm{Br}$ (Brown). In this picture, hydrogen atoms are omitted for clarity. 

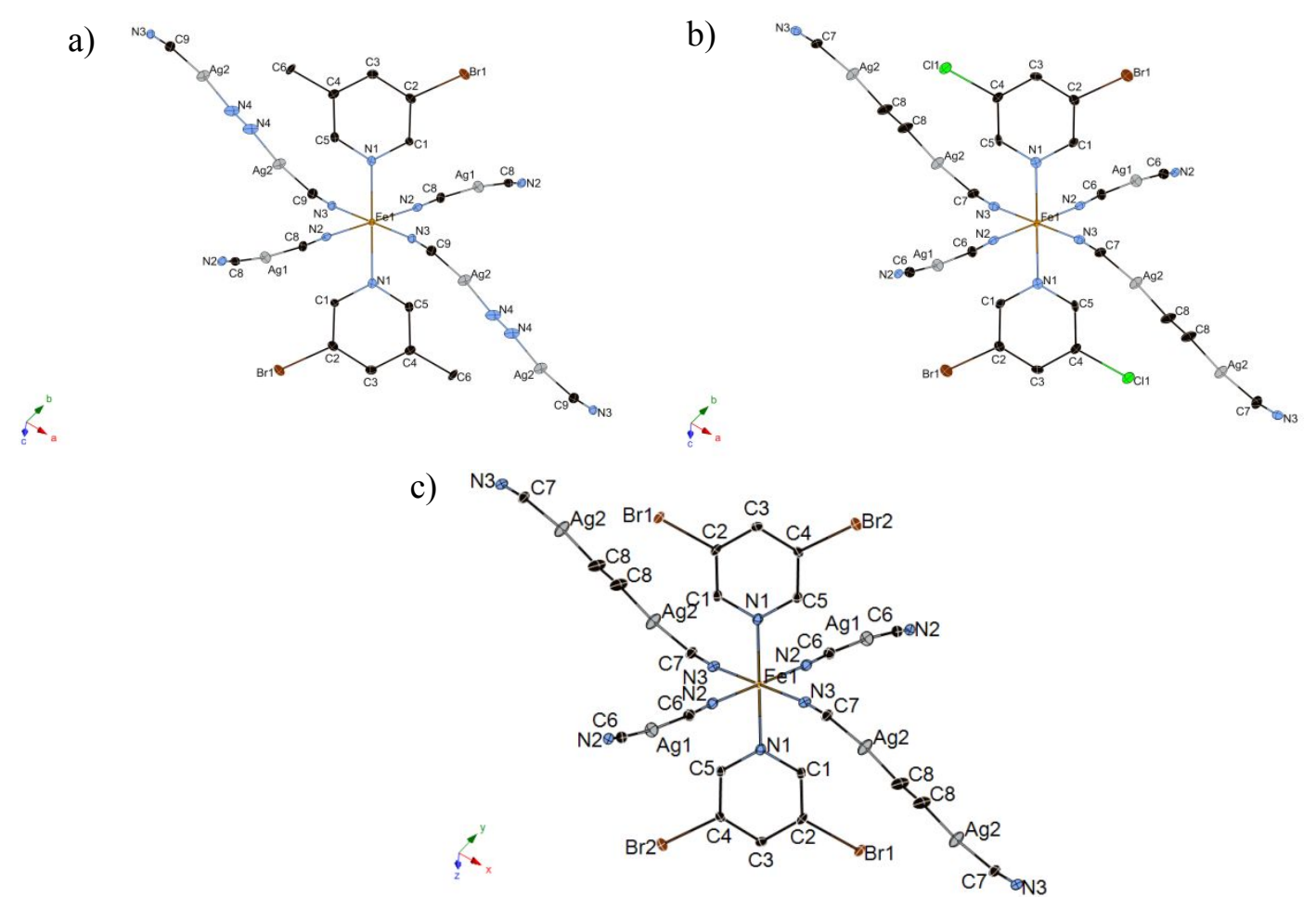

Figure S3. Molecular fragment of 1 at $90 \mathrm{~K}$ (a), 2 at $90 \mathrm{~K}$ (b), 3 at $90 \mathrm{~K}$ (c). In these pictures, hydrogen atoms are omitted for clarity. 
Table S1. Selected bond lengths and angles of 1-3.

\begin{tabular}{|c|c|c|c|}
\hline $1(293 \mathrm{~K})$ & & $1(90 \mathrm{~K})$ & \\
\hline Bond lengths $(\AA)$ & Bond Angles $\left({ }^{\circ}\right)$ & Bond lengths $(\AA)$ & Bond Angles $\left({ }^{\circ}\right)$ \\
\hline $\mathrm{Fe}(1)-\mathrm{N}(1): 2.217(5)$ & $\mathrm{N}(1)-\mathrm{Fe}(1)-\mathrm{N}(1): 180.0$ & $\mathrm{Fe}(1)-\mathrm{N}(1): 1.997(3)$ & $\mathrm{N}(1)-\mathrm{Fe}(1)-\mathrm{N}(1): 180.0$ \\
\hline $\mathrm{Fe}(1)-\mathrm{N}(2): 2.143(5)$ & $\mathrm{N}(2)-\mathrm{Fe}(1)-\mathrm{N}(1): 90.47(19)$ & $\mathrm{Fe}(1)-\mathrm{N}(2): 1.931(3)$ & $\mathrm{N}(2)-\mathrm{Fe}(1)-\mathrm{N}(1): 90.13(12)$ \\
\hline $\mathrm{Fe}(1)-\mathrm{N}(3): 2.133(5)$ & $\mathrm{N}(3)-\mathrm{Fe}(1)-\mathrm{N}(1): 89.90(19)$ & $\mathrm{Fe}(1)-\mathrm{N}(3): 1.940(3)$ & $\mathrm{N}(3)-\mathrm{Fe}(1)-\mathrm{N}(1): 89.90(12)$ \\
\hline $\operatorname{Ag}(1)-C(7): 2.058(6)$ & $\mathrm{C}(7)-\mathrm{Ag}(1)-\mathrm{C}(7): 171.5(3)$ & $\operatorname{Ag}(1)-C(8): 2.055(3)$ & $\mathrm{C}(8)-\mathrm{Ag}(1)-\mathrm{C}(8): 170.0(2)$ \\
\hline $\operatorname{Ag}(2)-C(8): 2.034(6)$ & $\mathrm{C}(8)-\mathrm{Ag}(2)-\mathrm{C}(9): 174.7(3)$ & $\operatorname{Ag}(2)-C(9): 2.053(6)$ & $\mathrm{C}(9)-\operatorname{Ag}(2)-\mathrm{N}(4): 174.03(15)$ \\
\hline $\mathrm{Ag}(2)-\mathrm{C}(9): 2.061(6)$ & & $\operatorname{Ag}(2)-N(4): 2.072(6)$ & \\
\hline $2(275 \mathrm{~K})$ & & $2(90 \mathrm{~K})$ & \\
\hline Bond lengths $(\AA)$ & Bond Angles $\left({ }^{\circ}\right)$ & Bond lengths $(\AA)$ & Bond Angles $\left({ }^{\circ}\right)$ \\
\hline $\mathrm{Fe}(1)-\mathrm{N}(1): 2.213(5)$ & $\mathrm{N}(1)-\mathrm{Fe}(1)-\mathrm{N}(1): 180.0$ & $\mathrm{Fe}(1)-\mathrm{N}(1): 1.995(3)$ & $\mathrm{N}(1)-\mathrm{Fe}(1)-\mathrm{N}(1): 180.0(2)$ \\
\hline $\mathrm{Fe}(1)-\mathrm{N}(2): 2.123(5)$ & $\mathrm{N}(2)-\mathrm{Fe}(1)-\mathrm{N}(1): 90.1(2)$ & $\mathrm{Fe}(1)-\mathrm{N}(2): 1.939(3)$ & $\mathrm{N}(2)-\mathrm{Fe}(1)-\mathrm{N}(1): 90.12(11)$ \\
\hline $\mathrm{Fe}(1)-\mathrm{N}(3): 2.139(5)$ & $\mathrm{N}(3)-\mathrm{Fe}(1)-\mathrm{N}(1): 90.69(19)$ & $\mathrm{Fe}(1)-\mathrm{N}(3): 1.938(3)$ & $\mathrm{N}(3)-\mathrm{Fe}(1)-\mathrm{N}(1): 89.91(11)$ \\
\hline $\operatorname{Ag}(1)-C(6): 2.054(7)$ & $C(6)-\operatorname{Ag}(1)-C(6): 171.4(4)$ & $\mathrm{Ag}(1)-\mathrm{C}(6): 2.054(3)$ & $\mathrm{C}(6)-\mathrm{Ag}(1)-\mathrm{C}(6): 170.83(18)$ \\
\hline $\operatorname{Ag}(2)-C(7): 2.054(7)$ & $C(7)-\operatorname{Ag}(2)-N(4): 176.1(3)$ & $\mathrm{Ag}(2)-\mathrm{C}(7): 2.050(3)$ & $\mathrm{C}(7)-\mathrm{Ag}(2)-\mathrm{C}(8): 174.75(13)$ \\
\hline $\mathrm{Ag}(2)-\mathrm{N}(4): 2.057(6)$ & & $\operatorname{Ag}(2)-C(8): 2.072(3)$ & \\
\hline $3(275 \mathrm{~K})$ & & $3(90 \mathrm{~K})$ & \\
\hline Bond lengths $(\AA)$ & Bond Angles $\left({ }^{\circ}\right)$ & Bond lengths $(\AA)$ & Bond Angles $\left({ }^{\circ}\right)$ \\
\hline $\mathrm{Fe}(1)-\mathrm{N}(1): 2.216(3)$ & $\mathrm{N}(1)-\mathrm{Fe}(1)-\mathrm{N}(1): 180.0$ & $\mathrm{Fe}(1)-\mathrm{N}(1): 2.013(3)$ & $\mathrm{N}(1)-\mathrm{Fe}(1)-\mathrm{N}(1): 180.0$ \\
\hline $\mathrm{Fe}(1)-\mathrm{N}(2): 2.121(3)$ & $\mathrm{N}(2)-\mathrm{Fe}(1)-\mathrm{N}(1): 90.02(13)$ & $\mathrm{Fe}(1)-\mathrm{N}(2): 1.948(3)$ & $\mathrm{N}(2)-\mathrm{Fe}(1)-\mathrm{N}(1): 90.01(10)$ \\
\hline $\mathrm{Fe}(1)-\mathrm{N}(3): 2.129(3)$ & $\mathrm{N}(3)-\mathrm{Fe}(1)-\mathrm{N}(1): 90.17(13)$ & $\mathrm{Fe}(1)-\mathrm{N}(3): 1.946(3)$ & $\mathrm{N}(3)-\mathrm{Fe}(1)-\mathrm{N}(1): 90.01(10)$ \\
\hline $\mathrm{Ag}(1)-\mathrm{C}(6): 2.053(4)$ & $C(6)-\operatorname{Ag}(1)-C(6): 171.4(2)$ & $\operatorname{Ag}(1)-C(6): 2.058(3)$ & $\mathrm{C}(6)-\mathrm{Ag}(1)-\mathrm{C}(6): 170.68(18)$ \\
\hline $\operatorname{Ag}(2)-C(7): 2.053(4)$ & $C(7)-\operatorname{Ag}(2)-C(8): 176.5(2)$ & $\mathrm{Ag}(2)-\mathrm{C}(7): 2.054(3)$ & $\mathrm{C}(7)-\operatorname{Ag}(2)-\mathrm{C}(8): 175.18(14)$ \\
\hline $\operatorname{Ag}(2)-C(8): 2.058(4)$ & & $\operatorname{Ag}(2)-C(8): 2.073(3)$ & \\
\hline
\end{tabular}




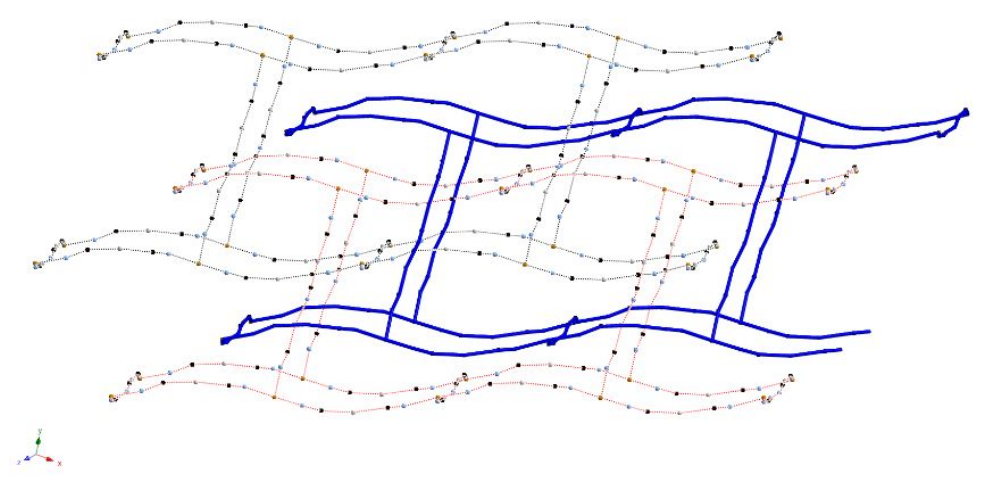

Figure S4. The drawing, triply interpenetrated 3D-networks. In this picture, pyridine ligands are omitted for clarity.

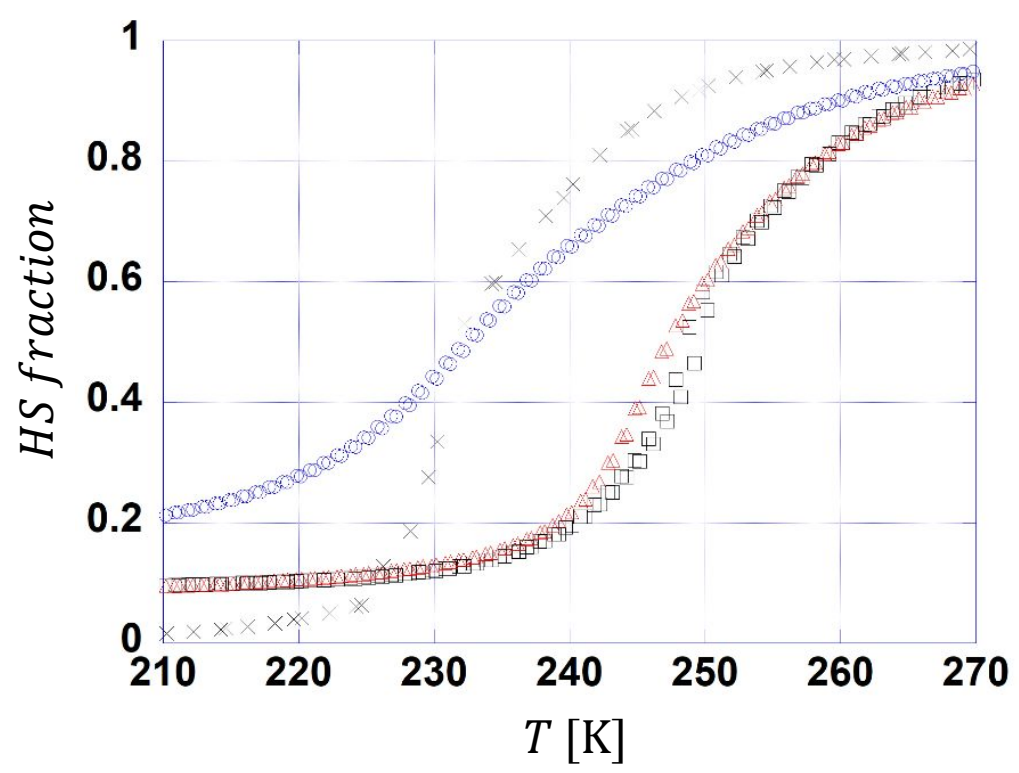

Figure S5. Thermal dependence of the HS fraction of 1 (blue), 2 (red), 3 (black) and 4 (black cross). 


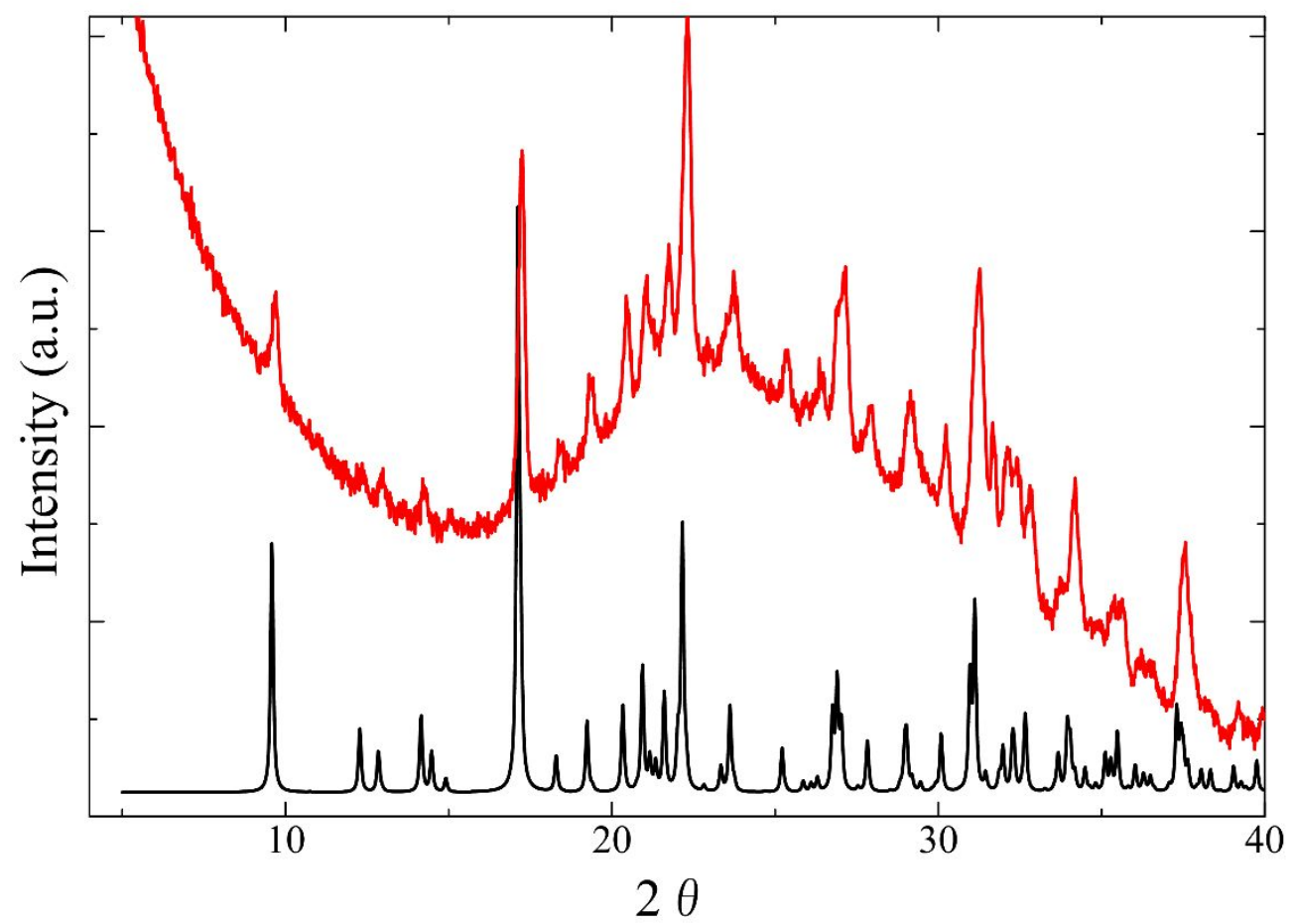

Figure S6. X-ray Powder diffraction for $\mathbf{1}$ (red line) and simulation pattern (black line).

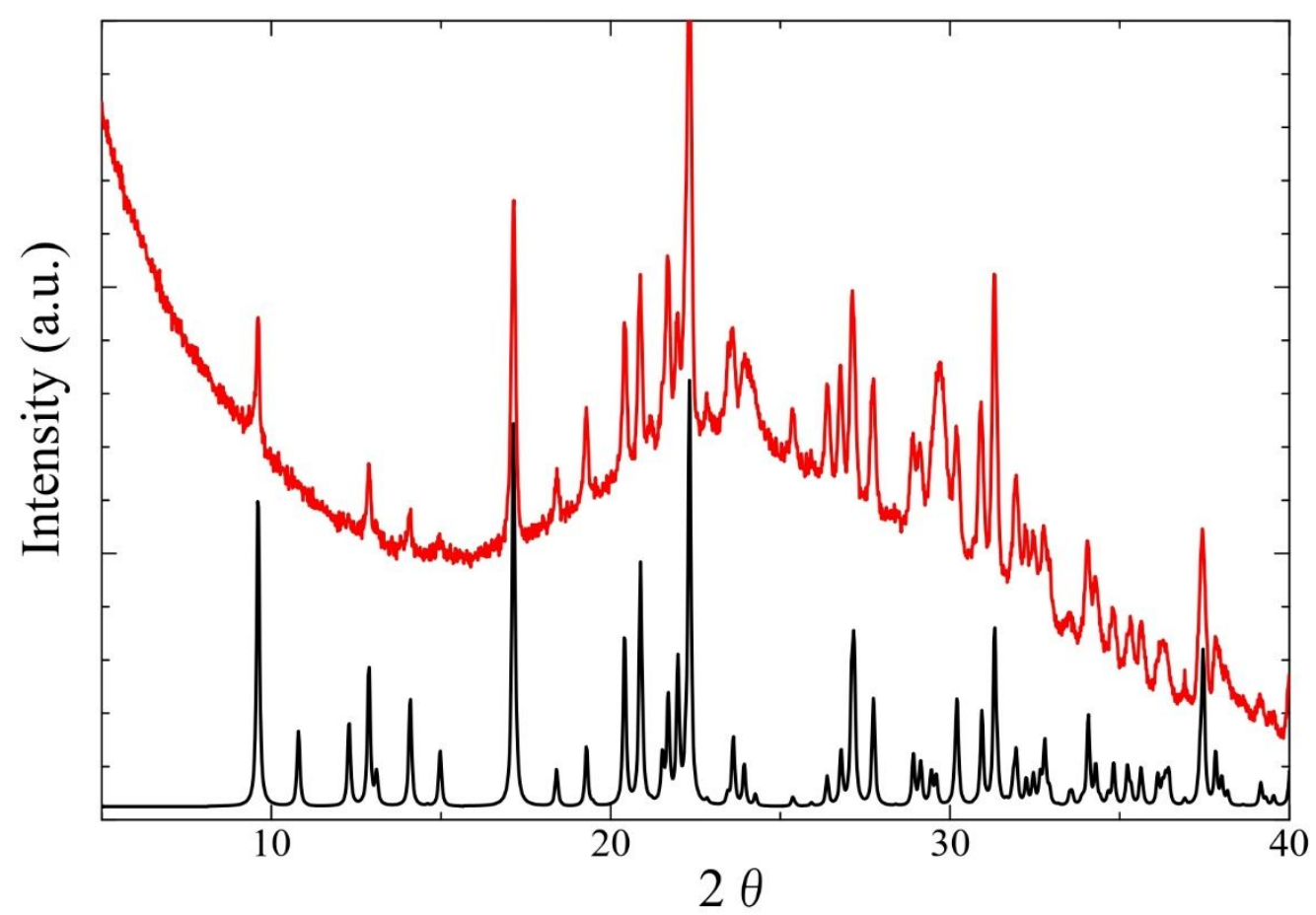

Figure S7. X-ray Powder diffraction for $\mathbf{2}$ (red line) and simulation pattern (black line). 


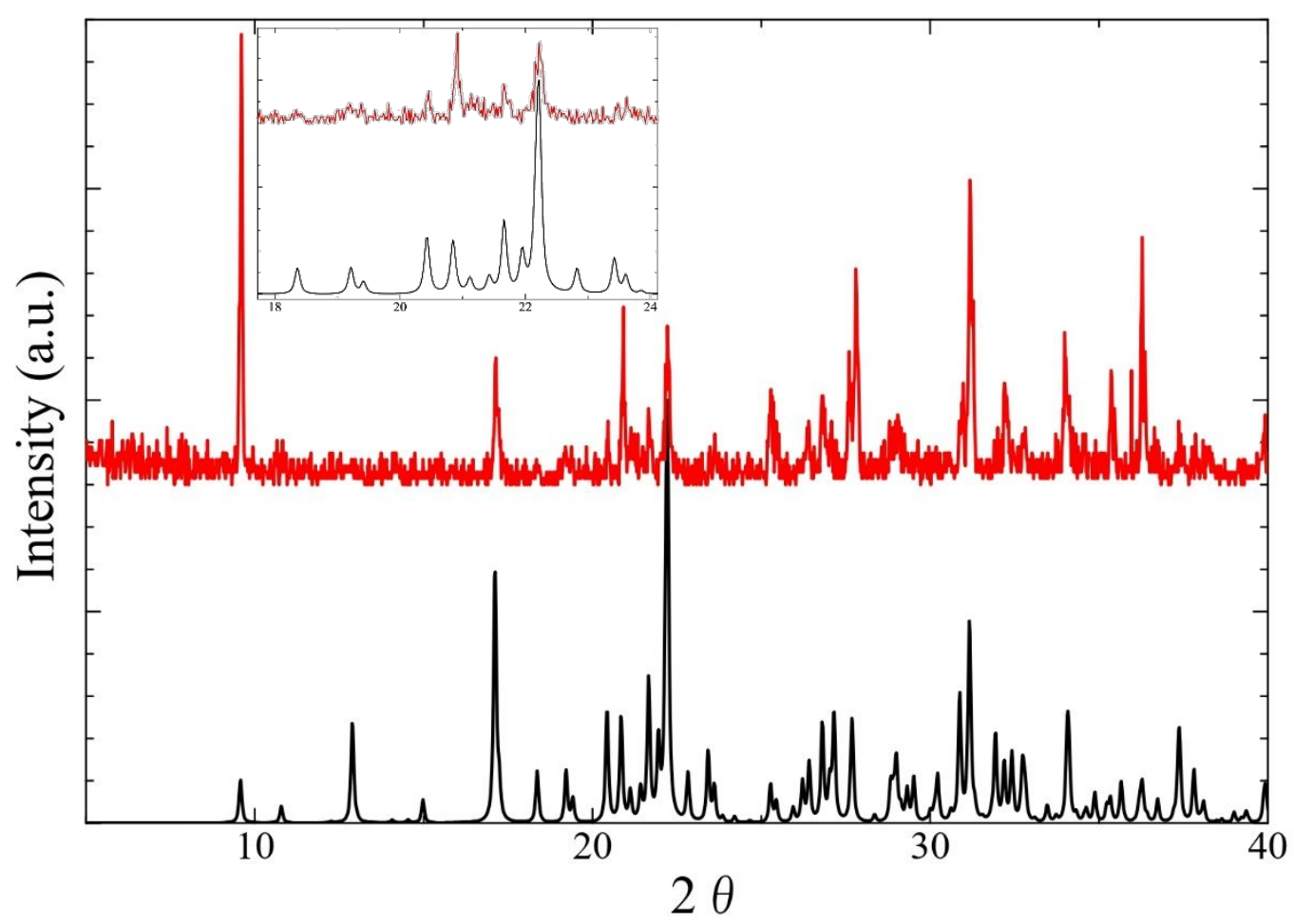

Figure S8. X-ray Powder diffraction for 3 (red line) and simulation pattern (black line). Cation: The intensities of the powder sample strongly depend on the orientation. 
Table S2 Crystal Data for 4 (ref 7)

\begin{tabular}{lll}
\hline Crystal data & $\mathbf{4}(293 \mathrm{~K})$ & $\mathbf{4}(80 \mathrm{~K})$ \\
\hline Empirical formula & $\mathrm{C}_{19} \mathrm{H}_{18} \mathrm{Ag}_{3} \mathrm{FeN}_{7}$ & $\mathrm{C}_{19} \mathrm{H}_{18} \mathrm{Ag}_{3} \mathrm{FeN}_{7}$ \\
FW & 723.86 & 723.86 \\
Temperature (K) & $293(2)$ & $80(2)$ \\
Crystal system & Monoclinic & Monoclinic \\
Space group & $C 2 / c$ & $C 2 / c$ \\
$a(\AA)$ & $12.7485(11)$ & $12.4686(10)$ \\
$b(\AA)$ & $13.5326(11)$ & $13.0951(10)$ \\
$c(\AA)$ & $14.0610(11)$ & $13.6139(11)$ \\
$\alpha\left(^{\circ}\right)$ & & \\
$\beta\left({ }^{\circ}\right)$ & $101.028(2)$ & $99.954(2)$ \\
$\gamma\left({ }^{\circ}\right)$ & & \\
$V\left(\AA^{3}\right)$ & $2381.0(3)$ & $2189.4(3)$ \\
$Z$ & 4 & 4 \\
\hline
\end{tabular}

